\title{
Finite-Time H-Infinity Filter Design for Discrete Jump Time-Delay Neural Networks
}

\author{
Yingqi Zhang*, Yang Yang, Caixia Liu and Jiankang Mu \\ College of Science, Henan University of Technology, Zhengzhou 450001, China \\ *Corresponding author
}

\begin{abstract}
This paper studies the problem of finite-time H-infinity filter design of discrete Markovian jump neural networks with time-varying delays and norm-bounded disturbance. The definitions of stochastic finite-time boundedness and stochastic $\mathrm{H}$-infinity finite-time boundedness are first given. Then, an $\mathrm{H}$ infinity state estimator is designed for the extended neural networks to ensure stochastic finite-time boundedness of the error dynamics with the prescribed disturbance attenuation level in a given finite-time interval. Furthermore, sufficient criteria are presented for the solvability of the state estimation problems by applying the linear matrix inequality technique and Markovian system approach. Finally, an example is presented to show the validity of the design method.
\end{abstract}

Keywords-neural networks; stochastic $h$-infinity finite-time boundedness; linear matrix inequalities (LMIs)

\section{INTRODUCTION}

In the past few decade, much attention has been paid to studying the stability analysis of time-delay neural networks and many results have been investigated due to a variety of their successful applications in various areas, including pattern recognition, signal processing, static image processing, associative memories, and target tracking [1-3]. In many practical applications, the information latching often happens in neural networks. Therefore, neural networks with Markovian jump have attracted a lot of research interests in mathematics and control communities, and much work has been studied in the literature, such as discrete-time neural networks [4-6] and continuous-time ones [7-10].

On the other hand, it should be noted that the considered neuron states are not fully available in real applications. Therefore, it is important and necessary to estimate the neuron state through available output measurements to make fully use of neural networks in practice. For instance, in [11], the problem of state estimation was investigated for delayed neural networks when the neuron activation function of the measurement nonlinearity satisfied the standard Lipschitz condition by using the LMI technique. The authors in [12] investigated the state estimation problem of neural networks with Markovian jump parameters and time delays under the same Lipschitz condition for the measurement nonlinearity. Recently, under the assumption that the neuron activation function and the nonlinearity satisfied the type of sectorbounded conditions, the problem of state estimation was addressed for continuous-time neural networks with Markovian jumps and distributed delays in [13]. More detailed results on the topic are referred the reader to [14-16].
As we know, the classical Lyapunov theory mainly deals with the asymptotic behavior of differential equations over infinite time interval and in general Lyapunov stability is enough for theoretical applications. However, the main attention in engineering applications is related to the behavior of the dynamics within a fixed finite time interval, for example, values of the state trajectories should be kept within some prescribed bounds in the presence of saturations in [17]. To tackle this transient performance of control systems, the finitetime stability or short-time stability was found in [18]. By utilizing linear matrix inequality (LMI) technique and Lyapunov function approach, a lot of interesting results on finite-time stability, finite-time boundedness and finite-time $\mathrm{H} \infty$ control have been obtained for stochastic systems [19]-[24], singular systems [22, 23], neural networks [20], and so on.

In this paper, the problem of finite-time $\mathrm{H} \infty$ filter design is investigated for discrete-time time-delay neural networks with Markovian jumps and norm-bounded disturbance. Firstly, the concepts of stochastic finite-time boundedness and stochastic $\mathrm{H} \infty$ finite-time boundedness are presented. Then, the $\mathrm{H} \infty$ filter is designed for the extended neural networks to ensure stochastic finite-time boundedness of the error dynamics with a prescribed performance level in the given finite-time interval. Sufficient criteria are also provided for the solvability of the state estimation problems by applying the linear matrix inequality technique and Markovian system approach. Finally, a numerical example is given to illustrate the validity of the obtained results.

\section{PROBLEM Formulation}

Consider the discrete-time Markovian jump neural network (DMJNN) with time-delays:

$$
\begin{aligned}
x(k+1)= & A\left(r_{k}\right) x(k)+B\left(r_{k}\right) f(x(k)) \\
& +C\left(r_{k}\right) g(x(k-h(k)))+B_{w}\left(r_{k}\right) w(k), \\
y(k)= & C_{y}\left(r_{k}\right) x(k), \\
z(k) \quad= & D\left(r_{k}\right) x(k)+E\left(r_{k}\right) w(k), \\
x(j) \quad= & \varphi(j), \quad j \in\left\{-h_{M}, \cdots,-1,0\right\},
\end{aligned}
$$

where $x(k)=\left[x_{1}(k), x_{2}(k), \cdots, x_{n}(k)\right]^{T} \in \mathbb{R}^{n}$ is the state vector of the neuron network, $y(k) \in \mathbb{R}^{m_{1}}$ is the measurement output, $z(k) \in \mathbb{R}^{m_{2}}$ is the signal to be estimated, $w(k) \in \mathbb{R}^{p}$ is the exogenous disturbance signal belonging to $l_{2}[0, \infty], \varphi(j)$ is a compatible vector-valued initial condition, $h(k)$ represents 
the transmission delay satisfying $0<h_{m} \leq h(k) \leq h_{M} \cdot f(x(k))$ and $g(x(k-h(k)))$ are the neuron activation functions. $A\left(r_{k}\right)=\operatorname{diag}\left(a_{1}\left(r_{k}\right), a_{2}\left(r_{k}\right), \cdots, a_{n}\left(r_{k}\right)\right)$ is the unknown modedependent diagonal matrix. The mode-dependent matrices $B\left(r_{k}\right), C\left(r_{k}\right), D\left(r_{k}\right), E\left(r_{k}\right)$ and $C_{y}\left(r_{k}\right)$ are the connection weight matrix and the gain matrix of the network measurement, respectively. The matrices are functions of the stochastic jump process $\left\{r_{k}, k \geq 0\right\}$, which is a discrete-time, discrete-state Markov chain taking values in a finite set $\Lambda=\{1,2, \cdots, s\}$ with transition probabilities

$$
\operatorname{Pr}\left\{r_{k+1}=j \mid r_{k}=i\right\}=\pi_{i j},
$$

where $\pi_{i j} \geq 0$ and $\sum_{j=1}^{S} \pi_{i j}=1$ for all $i \in \Lambda$.

For notational simplicity, $\mathbb{R}^{n}, \mathbb{R}^{n \times m}$ and $N^{+}$represent the sets of $n$ component real vectors, $n \times m$ real matrices and the set of nonnegative integers, respectively. $\mathrm{E}\{$.$\} denotes the$ expectation operator with respective to some probability measure. $M^{T}$ stands for the transpose of the matrix or vector $M$, the symbol * represents the transposed elements in the symmetric positions of a matrix. $I$ and $\operatorname{diag}(\cdots)$ stand for the identity of compatible dimension and a block-diagonal matrix, respectively. Moreover, for notational simplicity, for each possible $r_{k}=i, i \in \Lambda$, a matrix $G\left(r_{k}\right)$ will be denoted by $G_{i}$; for instance, $A\left(r_{k}\right)$ will be denoted by $A_{i}, B\left(r_{k}\right)$ by $B_{i}$, and so on.

In order to estimate the state of the neural network (1), we construct the filter of the following structure:

$$
\begin{aligned}
\tilde{x}(k+1)= & A_{f i} \tilde{x}(k)+B_{f i} f(\tilde{x}(k)) \\
& +C_{f i} g(\tilde{x}(k-h(k)))+H_{f i} y(k), \\
\tilde{z}(k) \quad= & D_{f i} \tilde{x}(k), \\
x(j) \quad= & \phi(j), \quad j \in\left\{-h_{M}, \cdots,-1,0\right\},
\end{aligned}
$$

where $\tilde{x}(k) \in \mathbb{R}^{n}$ is the filter state, $\tilde{z}(k) \in \mathbb{R}^{m_{2}}$ is the filter output, $\phi(j)$ is a compatible filter vector-valued initial condition, $A_{f i}, B_{f i}, C_{f i}, D_{f i}$ and $H_{f i}$ are to design the filtering matrices with appropriate dimensions.

Define

$$
\bar{z}(k)=z(k)-\tilde{z}(k) \text { and } \bar{x}^{T}(k)=\left[x^{T}(k) x^{T}(k)-\tilde{x}^{T}(k)\right] .
$$

Combining the DMJNN (1) with the filter system (3), we can obtain the following extend time-delay DMJNN:

$$
\begin{aligned}
\bar{x}(k+1)= & \bar{A}_{i} \bar{x}(k)+\bar{B}_{i} F(\bar{x}(k)) \\
& +\bar{C}_{i} F(\bar{x}(k-h(k)))+\bar{B}_{w i} w(k),
\end{aligned}
$$

$$
\tilde{z}(k) \quad=\bar{D}_{i} \bar{x}(k)+E_{i} w(k)
$$

where

$$
\begin{gathered}
\bar{A}_{i}=\left[\begin{array}{cc}
A_{i} & 0 \\
A_{i}-A_{f i}-H_{f i} C_{y i} & A_{f i}
\end{array}\right], \bar{D}_{i}=\left[\begin{array}{cc}
D_{i}-D_{f i} & D_{f i}
\end{array}\right], \\
\bar{B}_{i}=\left[\begin{array}{cc}
B_{i} & 0 \\
B_{i}-B_{f i} & B_{f i}
\end{array}\right], \bar{C}_{i}=\left[\begin{array}{cc}
C_{i} & 0 \\
C_{i}-C_{f i} & C_{f i}
\end{array}\right], \bar{B}_{w i}=\left[\begin{array}{l}
B_{w i} \\
B_{w i}
\end{array}\right], \\
F^{T}(\bar{x}(k))=\left[\begin{array}{ll}
f^{T}(x(k)) & f^{T}(x(k))-f^{T}(\tilde{x}(k))
\end{array}\right], \\
G^{T}(\bar{x}(k))=\left[\begin{array}{ll}
g^{T}(x(k)) & g^{T}(x(k))-g^{T}(\tilde{x}(k))
\end{array}\right] .
\end{gathered}
$$

For the neuron activation functions and disturbance input, we have the following assumptions.

Assumption 1. The neuron state-based nonlinear functions $f(\cdot)$ and $g(\cdot)$ in (1) are continuous and satisfy $f(0)=0$, $g(0)=0$ and the following sector-bounded conditions

$$
\begin{aligned}
& {\left[f(x, y)-U_{1}(x-y)\right]^{T}\left[f(x, y)-U_{2}(x-y)\right] \leq 0,} \\
& {\left[g(x, y)-V_{1}(x-y)\right]^{T}\left[g(x, y)-V_{2}(x-y)\right] \leq 0,}
\end{aligned}
$$

where $f(x, y)=f(x)-f(y), g(x, y)=g(x)-g(y), U_{1}, U_{2}$, $V_{1}$ and $V_{2}$ are real matrices of appropriate dimensions.

Assumption 2. The disturbance input $w(k) \in \mathbb{R}^{p}$ satisfies

$$
\mathrm{E}\left\{\sum_{k=0}^{\infty} w^{T}(k) w(k)\right\} \leq \delta^{2}, \delta \geq 0 .
$$

Throughout the paper, we need the following definitions.

Definition 1 (SFTS). The time-delay DMJNN (1) with $w(k)=0$ is said to be stochastically finite-time stable (SFTS) with respect to $\left(c_{1}, c_{2}, R_{i}, N\right)$, where $0<c_{1}<c_{2}, R_{i}>0$ and $N \in \mathbb{N}^{+}$, if

$$
\begin{gathered}
\mathrm{E}\left\{x^{T}\left(k_{1}\right) R_{i} x\left(k_{2}\right)\right\} \leq c_{1}^{2} \Rightarrow \mathrm{E}\left\{x^{T}\left(k_{1}\right) R_{i} x\left(k_{2}\right)\right\}<c_{2}^{2}, \\
\forall k_{1} \in\left\{-h_{M}, \cdots,-1,0\right\}, \forall k_{2} \in\{1,2, \cdots, n\} .
\end{gathered}
$$

Definition 2 (SFTB). The extended time-delay DMJNN (4) is said to be stochastically finite-time bounded (SFTB) with respect to $\left(c_{1}, c_{2}, \bar{R}_{i}, N, \delta\right)$, where $0<c_{1}<c_{2}, \bar{R}_{i}>0$ and $N \in \mathbb{N}^{+}$, if

$$
\begin{gathered}
\mathrm{E}\left\{\bar{x}^{T}\left(k_{1}\right) \bar{R}_{i} x\left(k_{2}\right)\right\} \leq c_{1}^{2} \Rightarrow \mathrm{E}\left\{\bar{x}^{T}\left(k_{1}\right) \bar{R}_{i} \bar{x}\left(k_{2}\right)\right\}<c_{2}^{2}, \\
\forall k_{1} \in\left\{-h_{M}, \cdots,-1,0\right\}, \forall k_{2} \in\{1,2, \cdots, n\} .
\end{gathered}
$$


Definition 3 (stochastic finite-time $\mathrm{H} \infty$ state estimation). The extended time-delay DMJNN (4) is said to be stochastically $\mathrm{H} \infty$ finite-time bounded with respect to $\left(c_{1}, c_{2}, \bar{R}_{i}, N, \delta, \gamma\right)$, where $0<c_{1}<c_{2}, \bar{R}_{i}>0, \gamma>0$ and $N \in \mathbb{N}^{+}$, if the filtering error DMJNN (4) is SFTB with respect to $\left(c_{1}, c_{2}, \bar{R}_{i}, N, \delta\right)$, and under the zero-initial condition the filtering error $\bar{Z}(k)$ satisfies

$$
\mathrm{E}\left\{\sum_{k=0}^{N} \bar{Z}^{T}(k) \bar{Z}(k)\right\}<\gamma^{2} \mathrm{E}\left\{\sum_{k=0}^{N} w^{T}(k) w(k)\right\} .
$$

Moreover, the filter (3) is called the finite-time $\mathrm{H} \infty$ filter of the extended time-delay DMJNN (4).

\section{MAIN RESULTS}

In this section, we give our main results and the proofs of the results are also omitted due to the space limit.

Theorem 1. For given $c_{1}>0, \bar{R}_{i}>0, N \in \mathbb{N}^{+}, \delta \geq 0$ and $\mu \geq 1$, under Assumptions 1 and 2, the extended time-delay DMJNN (4) is SFTB with respect to $\left(c_{1}, c_{2}, \bar{R}_{i}, N, \delta\right)$, if positive scalars $c_{2}>0, \eta_{1}>0, \eta_{2}>0, \eta_{3}>0$, sets of positive scalars $\left\{\sigma_{1 i}, i \in \Lambda\right\}$ and $\left\{\sigma_{2 i}, i \in \Lambda\right\}$, sets of symmetric positive-definite matrices $\left\{P_{1 i}, i \in \Lambda\right\} \quad\left\{P_{2 i}, i \in \Lambda\right\} \quad$ and $\left\{Q_{i}, i \in \Lambda\right\}$, sets of matrices $\left\{W_{i}, i \in \Lambda\right\},\left\{X_{i}, i \in \Lambda\right\}$, $\left\{Y_{i}, i \in \Lambda\right\}, \quad\left\{Z_{i}, i \in \Lambda\right\}$, and a symmetric positive-definite matrix $Q$, for all $i \in \Lambda$, such that

$$
\begin{gathered}
{\left[\begin{array}{cc}
\Lambda_{11 i} & * \\
\Lambda_{21 i} & \Lambda_{22 i}
\end{array}\right]<0,} \\
\bar{R}_{i}<\bar{P}_{i}<\eta_{1} \bar{R}_{i}, \\
0<Q<\eta_{2} \bar{R}_{i}, \\
0<Q_{i}<\eta_{3} I, \\
\left(\eta_{1}+\bar{h} \eta_{2}\right) c_{1}^{2}+\eta_{3} \delta^{2}<\mu^{-N} c_{2}^{2},
\end{gathered}
$$

where

$$
\begin{aligned}
& \bar{P}_{i}=\operatorname{diag}\left\{P_{1 i}, P_{2 i}\right\}, \bar{R}_{i}=\operatorname{diag}\left\{R_{1 i}, R_{2 i}\right\}, \\
& \Lambda_{22 i}=\operatorname{diag}\left\{\bar{P}_{i}-2 \bar{P}_{1}, \bar{P}_{i}-2 \bar{P}_{2}, \cdots, \bar{P}_{i}-2 \bar{P}_{S}\right\}, \\
& \bar{h}=h_{M}+\left(h_{M}-h_{m}\right)\left(h_{M}+h_{m}-1\right) / 2, \\
& \Lambda_{21 i}=\tilde{\pi}_{i}^{T}\left[\begin{array}{lllll}
L_{1 i} & 0 & L_{2 i} & L_{3 i} & L_{4 i}
\end{array}\right], \\
& \tilde{\pi}_{i}=\left[\begin{array}{llll}
\sqrt{\pi_{i 1}} I & \sqrt{\pi_{i 2}} I & \cdots & \sqrt{\pi_{i S}} I
\end{array}\right],
\end{aligned}
$$

$$
\Lambda_{11 i}=\left[\begin{array}{ccccc}
\Lambda_{11 i}^{11} & * & * & * & * \\
0 & \Lambda_{11 i}^{22} & * & * & * \\
-\sigma_{1 i} F_{2} & 0 & -\sigma_{1 i} I & * & * \\
0 & -\sigma_{2 i} G_{2} & 0 & -\sigma_{2 i} I & * \\
0 & 0 & 0 & 0 & -Q_{i}
\end{array}\right]
$$

with $\Lambda_{11 i}^{11}=\left(h_{M}-h_{m}+1\right) Q-\mu \bar{P}_{i}-\sigma_{1 i} F_{1}, \Lambda_{11 i}^{22}=-Q-\sigma_{2 i} G_{1}$,

$$
\begin{gathered}
L_{1 i}=\left[\begin{array}{cc}
P_{1 i} A_{i} & 0 \\
P_{2 i} A_{i}-W_{i}-Z_{i} C_{y i} & W_{i}
\end{array}\right], L_{2 i}=\left[\begin{array}{cc}
P_{1 i} B_{i} & 0 \\
P_{2 i} B_{i}-X_{i} & X_{i}
\end{array}\right], \\
L_{3 i}=\left[\begin{array}{cc}
P_{1 i} C_{i} & 0 \\
P_{2 i} C_{i}-Y_{i} & Y_{i}
\end{array}\right], L_{4 i}=\left[\begin{array}{l}
P_{1 i} B_{w i} \\
P_{2 i} B_{w i}
\end{array}\right],
\end{gathered}
$$

$F_{1}=\left[\begin{array}{cc}\left(U_{1}^{T} U_{2}+U_{1} U_{2}^{T}\right) / 2 & * \\ 0 & \left(U_{1}^{T} U_{2}+U_{1} U_{2}^{T}\right) / 2\end{array}\right], F_{2}=\left[\begin{array}{cc}-\left(U_{1}+U_{2}\right) / 2 & * \\ 0 & -\left(U_{1}+U_{2}\right) / 2\end{array}\right]$,

$$
G_{1}=\left[\begin{array}{cc}
\left(V_{1}^{T} V_{2}+V_{1} V_{2}^{T}\right) / 2 & * \\
0 & \left(V_{1}^{T} V_{2}+V_{1} V_{2}^{T}\right) / 2
\end{array}\right], G_{2}=\left[\begin{array}{cc}
-\left(V_{1}+V_{2}\right) / 2 & * \\
0 & -\left(V_{1}+V_{2}\right) / 2
\end{array}\right] \text {. }
$$

Moreover, the desired state estimator gain matrices are given by

$$
A_{f i}=P_{2 i}^{-1} W_{i}, B_{f i}=P_{2 i}^{-1} X_{i}, C_{f i}=P_{2 i}^{-1} Y_{i}, H_{f i}=P_{2 i}^{-1} Z_{i}
$$

Theorem 2. For given $c_{1}>0, \bar{R}_{i}>0, N \in \mathbb{N}^{+}, \delta \geq 0$ and $\mu \geq 1$, under Assumptions 1 and 2, the extended time-delay DMJNN (4) is stochastically $\mathrm{H}_{\infty}$ finite-time bounded with respect to $\left(c_{1}, c_{2}, \bar{R}_{i}, N, \delta, \gamma\right)$, if there exist scalars $\mu \geq 1, c_{2}>0$, $\eta_{1}>0, \eta_{2}>0, \gamma>0$, sets of positive scalars $\left\{\sigma_{1 i}, i \in \Lambda\right\}$ and $\left\{\sigma_{2 i}, i \in \Lambda\right\}$, sets of symmetric positive-definite matrices $\left\{P_{1 i}, i \in \Lambda\right\} \quad$ and $\quad\left\{P_{2 i}, i \in \Lambda\right\} \quad$,sets of matrices $\left\{W_{i}, i \in \Lambda\right\},\left\{X_{i}, \quad i \in \Lambda\right\},\left\{Y_{i}, i \in \Lambda\right\},\left\{Z_{i}, i \in \Lambda\right\}$ and a symmetric positive-definite matrix $Q$, for all $i \in \Lambda$, such that (11b), (11c) and the following inequalities are satisfied:

$$
\begin{aligned}
& {\left[\begin{array}{ccc}
\tilde{\Lambda}_{11 i} & * & * \\
\Lambda_{21 i} & \Lambda_{22 i} & * \\
\Lambda_{31 i} & 0 & -I
\end{array}\right]<0,} \\
& \left(\eta_{1}+\bar{h} \eta_{2}\right) c_{1}^{2}+\gamma^{2} \mu^{-N} \delta^{2}<\mu^{-N} c_{2}^{2},
\end{aligned}
$$

where $\tilde{\Lambda}_{11 i}=\Lambda_{11 i}+\operatorname{diag}\left\{0,0,0,0, Q_{i}-\gamma^{2} \mu^{-N} I\right\}$, $\Lambda_{31 i}=\left[\begin{array}{llllll}D_{i}-D_{f i} & D_{f i} & 0 & 0 & 0 & E_{i}\end{array}\right]$ and other matrix parameters are defined in Theorem 1. Moreover, the desired filter gain matrices are given by

$$
A_{f i}=P_{2 i}^{-1} W_{i}, B_{f i}=P_{2 i}^{-1} X_{i}, C_{f i}=P_{2 i}^{-1} Y_{i}, H_{f i}=P_{2 i}^{-1} Z_{i} .
$$




\section{A NUMERICAL EXAMPLE}

In this section, we provide a simulation example to verify the developed results in this paper.

Example 1. To show stochastic $\mathrm{H} \infty$ finite-time boundedness for the extended time-delay DMJNN (4), we consider a time-delay neural network (1) with two jump modes and the following parameters:

\section{Mode 1:}

$$
\begin{aligned}
& A=\left[\begin{array}{ccc}
0.7 & 0 & 0 \\
0 & 0.8 & 0 \\
0 & 0 & 0.8
\end{array}\right], B_{2}=\left[\begin{array}{ccc}
0.3 & 0 & 0.4 \\
0.2 & -0.4 & 0.2 \\
0.3 & 0 & -0.5
\end{array}\right], C_{1}=\left[\begin{array}{ccc}
-0.15 & 0.3 & 0 \\
0.2 & 0.1 & 0.4 \\
0.1 & -0.2 & 0.3
\end{array}\right], \\
& D=\left[\begin{array}{ccc}
0.4 & 0.4 & -0.1 \\
0.2 & 0.4 & 0.2
\end{array}\right], C_{11}=\left[\begin{array}{ccc}
0.1 & -0.1 & 0.1 \\
0 & 0 & 0 \\
0 & 0.2 & 0.1
\end{array}\right], U_{1}=\left[\begin{array}{lll}
0 & 0.1 & 0.1 \\
0 & 0.2 & 0.1 \\
0 & 0.1 & 0.1
\end{array}\right], \\
& U_{2}=\left[\begin{array}{ccc}
0.1 & -0.1 & 0.1 \\
0 & 0 & 0 \\
0 & 0.2 & 0.1
\end{array}\right], B_{w 1}=\left[\begin{array}{c}
0.1 \\
0 \\
0.1
\end{array}\right], E_{1}=\left[\begin{array}{l}
0.1 \\
0.1
\end{array}\right]
\end{aligned}
$$

Mode 2:

$$
\begin{gathered}
A_{2}=\left[\begin{array}{ccc}
0.5 & 0 & 0 \\
0 & 0.75 & 0 \\
0 & 0 & 0.6
\end{array}\right], B_{2}=\left[\begin{array}{ccc}
0.4 & 0 & 0.3 \\
0.1 & -0.2 & 0.1 \\
0.2 & 0.1 & -0.3
\end{array}\right], C_{2}=\left[\begin{array}{ccc}
-0.2 & 0.1 & 0 \\
0.3 & 0 & 0.3 \\
0.2 & 0.1 & 0.15
\end{array}\right], \\
D_{2}=\left[\begin{array}{ccc}
0.2 & 0.1 & -0.1 \\
0.2 & 0.1 & 0.3
\end{array}\right], C_{y_{2}}=\left[\begin{array}{ccc}
0.1 & -0.15 & 0.1 \\
0.2 & 0 & 0.2 \\
0.1 & 0 & 0.2
\end{array}\right], V_{1}=\left[\begin{array}{ccc}
0 & 0.2 & 0 \\
0 & 0.3 & 0 \\
0.1 & 0 & 0.2
\end{array}\right], \\
V_{2}=\left[\begin{array}{ccc}
0.1 & -0.15 & 0.1 \\
0.2 & 0 & 0 \\
-0.1 & 0.2 & 0.2
\end{array}\right], B_{w 2}=\left[\begin{array}{c}
0.1 \\
0 \\
0.1
\end{array}\right], E_{2}=\left[\begin{array}{l}
0.3 \\
0.2
\end{array}\right] .
\end{gathered}
$$

In addition, the transition rate matrix is chosen by $\Pi=\left[\begin{array}{cc}0.7 & 0.3 \\ 0.35 & 0.65\end{array}\right]$.

Now, we assume the time-varying delay satisfy $1 \leq h(k) \leq 2$. Given the initial values for $R_{1}=R_{2}=I_{3}, c_{1}=1, N=5, \delta=2$ and $\mu=2$. Then, applying the Matlab LMI Control Toolbox to solve the feasibility problem in Theorem 2, the Desired filter gain matrices can be derived by

$$
A_{f 1}=\left[\begin{array}{ccc}
0.0032 & 0.0360 & 0.0073 \\
-0.0032 & -0.0762 & -0.0217 \\
0.0026 & -0.1258 & -0.0464
\end{array}\right], A_{f 2}=\left[\begin{array}{ccc}
-0.0148 & 0.0307 & 0.0071 \\
-0.0033 & -0.0321 & 0.0058 \\
-0.0009 & 0.0029 & -0.0230
\end{array}\right],
$$

$$
\begin{aligned}
& B_{f 1}=\left[\begin{array}{ccc}
0.0052 & -0.0253 & -0.1034 \\
-0.0055 & 0.0575 & 0.3482 \\
-0.0006 & 0.1506 & 0.7893
\end{array}\right], B_{f 2}=\left[\begin{array}{ccc}
0.2321 & -0.2811 & -0.0464 \\
-0.0149 & 0.2204 & 0.0296 \\
0.2359 & 0.7079 & -0.4030
\end{array}\right], \\
& C_{f 1}=\left[\begin{array}{ccc}
0.0086 & 0.2475 & -0.0971 \\
-0.0267 & -0.1875 & 0.1538 \\
0.1820 & 0.0607 & 0.3414
\end{array}\right], C_{f 2}=\left[\begin{array}{ccc}
-0.0010 & 0.0028 & 0.0821 \\
-0.0009 & 0.0027 & 0.0565 \\
0.0235 & -0.0688 & 0.0028
\end{array}\right], \\
& H_{f 1}=\left[\begin{array}{ccc}
0.0350 & -0.1664 & -0.1506 \\
0.0072 & 0.0139 & 0.0126 \\
-0.1864 & 0.0284 & 0.0257
\end{array}\right], H_{f 2}=\left[\begin{array}{ccc}
-0.0686 & 0 & 0.0004 \\
0.0087 & 0 & 0.0001 \\
0.0112 & 0 & -0.0023
\end{array}\right], \\
& D_{f 1}=\left[\begin{array}{ccc}
0.0039 & -0.0004 & -0.0067 \\
-0.0041 & 0.0128 & 0.0925
\end{array}\right], \quad D_{f 2}=\left[\begin{array}{ccc}
0.1069 & 0.0722 & 0.0471 \\
0.0783 & 0.0592 & -0.0123
\end{array}\right],
\end{aligned}
$$

and $\gamma=90.4585$ and $c_{2}=211.6378$. Therefore, the error dynamics (4) is stochastically $\mathrm{H} \infty$ finite-time bounded with respect to $\left(1,211.6378, I_{6}, 5,2,90.4585\right)$.

\section{CONCLUSIONS}

This paper investigated the problem of finite-time $\mathrm{H} \infty$ filter design of discrete-time, time-delay neural networks with Markovian jump parameters and norm-bounded disturbance. Assumed that the activation functions satisfy the sector-like nonlinearities, a state estimator is designed to guarantee stochastic finite-time boundedness of the resulted error dynamics with an $\mathrm{H} \infty$ performance level by using the stochastic Lyapunov-Krasovski functional approach and LMI technique. The results of a numerical example verify the effectiveness of the presented solutions.

\section{REFERENCES}

[1] S. Arik, "Global asymptotic stability of a class of dynamical neural networks," IEEE Trans Circuits Syst. I, Fundam. Theory Appl., vol. 47, no. 4, pp. 568-571, Apr. 2000.

[2] H. Karimi, H. Gao, and X. Yu, "New delay-dependent exponential Hळ synchronization for uncertain neural networks with mixed time delays," IEEE Trans. Syst. man Cybern., vol. 40, no. 1, pp. 173-185, Oct. 2010.

[3] Z. Wu, P. Shi, H. Su, and J. Chu, "Exponential synchronization of neural networks with discrete and distributed delays under time-varying sampling," IEEE Trans. Neural Netw. Learn. Syst., vol. 23, no. 9, pp.1368-1376, Sep.2012.

[4] P. Tino, M. Cernansky, and L. Benuskova, "Markovian architectural bias of recurrent neural networks," IEEE Trans. Neural Networks, vol. 15, no. 1, pp. 6-15, Jan. 2004

[5] Y. Liu, Z. Wang, J. Liang, and X. Liu, "Stability and synchronization of discrete-time Markovian jumping neural networks with mixed mode dependent time delays," IEEE Trans. Neural Netw., vol. 20, no. 7, pp.1102-1116, Jul. 2009.

[6] Z. Wang, Y. Liu, L. Yu, and X. Liu, "Exponential stability of delayed recurrent neural networks with Markovian jumping parameters," Phys. Lett. A., vol. 356, no. 4/5, pp. 346-352, Aug. 2006.

[7] Z. Wang, Y. Liu, M. Li, and X. Liu, "Stability analysis for stochastic Cohen-Grossberg neural networks with mixed time delays," IEEE Trans. Neural Netw., vol. 17, no. 3, pp. 814-820, Mar. 2006.

[8] H. Zhang and Y. Wang, "Stability analysis of Markovian jumping stochastic Cohen-Grossberg neural networks with mixed time delays," IEEE Trans. Neural Netw., vol. 19, no. 2, pp. 366-370, Feb. 2008.

[9] Z. Wu, P. Shi, H. Su, and J Chu, "Passivity analysis for discrete-time stochastic Markovian jump neural networks with mixed time-delays," IEEE Trans. Neural Netw., vol. 22, no. 10, pp. 1566-1575, Oct. 2011. 
[10] Z. Wu, P. Shi, H. Su, and J Chu, "Stochastic synchronization of Markovian jump neural networks with time-varying delay using sampled data," IEEE Trans. Cybern., vol. 43, no. 6, pp. 1796-1806, Dec. 2013.

[11] Z. Wang, D.W.C. Ho, and X. Liu, "State estimation for delayed neural networks," IEEE Trans. Neural Netw., vol. 16, no. 1, pp. 279-284, Jan. 2005.

[12] Z. Wang, Y. Liu, and X. Liu, "State estimation for jumping recurrent neural networks with discrete and distributed delays," Neural Netw., vol.22, no. 1, pp. 41-48, Jan. 2009.

[13] Y. Chen and W. Zheng, "Stochastic state estimation for neural networks with distributed delays and Markovian jump," Neural netw., vol. 25, pp.14-20, Jan. 2012.

[14] S. Lakshmanan, J. Park, D. Ji, H. Jung, and G. Nagamani, "State estimation of neural networks with time-varying delays and Markovian jumping parameter based on passivity theory," Nonlinear Dyn., vol. 70, no. 2, pp. 1421-1434, Feb. 2012.

[15] Z. Wu, P. Shi, H. Su, and J. Chu, "Dissipativity analysis for discretetime stochastic neural networks with time-varying delays," IEEE Trans. Neural Netw. Learn. Syst., vol. 24, no. 3 pp. 345-355, Mar. 2013.

[16] C.K. Ahn, "State estimation for T-S fuzzy Hopfield neural networks via strict output passivation of the error system," Int. J. Syst. Sci., vol. 42, no. 5, 503-518, July. 2013.

[17] F. Amato, M. Ariola, and P. Dorato, "Finite-time control of linear systems subject to parametric uncertainties and disturbances," Automatica, vol. 37, no. 9, pp. 1459-1463, Sep. 2001.

[18] F. Amato and M. Ariola, "Finite-time control of discrete-time linear systems," IEEE Trans. Autom. Control, vol. 50, no. 5, pp. 724-729, May 2005.

[19] Y. Zhang, C. Liu, and Y. Song, "Finite-time Hœ filtering for discretetime Markovian jump systems," J. Frankl. Inst., vol. 350, no. 6, pp. 1579-1595, Aug. 2013.

[20] Y. Zhang, P. Shi, S.K. Nguang, J. Zhang, and H.R. Karimi, "Finite-time boundedness for uncertain discrete neural networks with time-delays and Markovian jumps," Neurocomputing, vol. 140, no. 22, pp. 1-7, Sep.2014.

[21] X. Luan, F. Liu, and P. Shi, "Finite-time filtering for nonlinear stochastic systems with partially known transition jump rates," IET Control Theory Appl., vol. 4, no. 5, pp. 735-745, May 2010.

[22] Y. Zhang, C. Liu, and X. Mu, "Robust finite-time stabilization of uncertain singular Markovian jump systems," Appl. Math. Model., vol.36, no. 10, pp. 5109-5121, Oct. 2012.

[23] Y. Zhang, P. Shi, S.K. Nguang, and Y. Song, "Robust finite-time Hळ control for uncertain discrete-time singular systems with Markovian jumps," IET Control Theory Appl., vol. 8, no. 12, pp. 1105-1111, Aug.2014.

[24] Y. Zhang, G. Cheng, and C. Liu, "Finite-time unbiased Hळ filtering for discrete jump time-delay systems," Appl. Math. Model., vol. 38, no.13, pp. 3339-3349, Jul. 2014 